\title{
MOND and universality of average surface densities in galaxies
}

\author{
Gianfranco Gentile* \\ Sterrenkundig Observatorium, Universiteit Gent, Belgium \\ Astrophysical Institute, Vrije Universiteit Brussel, Belgium \\ E-mail: gianfranco.gentiledugent.be
}

Dark matter is one of the great mysteries of today's astrophysics. However, the dark matter particle has not been detected (yet?), so that in some astrophysical contexts its very existence is even being questioned. Therefore, it is worth investigating alternatives to galactic dark matter such as MOND (Modified Newtonian Dynamics). I discuss some pros and cons of dark matter and MOND to explain observational data, focussing on galaxy scales. I also discuss recent results on the universality of dark and baryonic average surface densities within one dark halo scale length in galaxies.

VIII International Workshop on the Dark Side of the Universe,

June 10-15, 2012

Rio de Janeiro, Brazil

${ }^{*}$ Speaker. 


\section{Introduction}

A very large number of astronomical observations, at the galactic, extragalactic, or cosmological level, point to the fact that either there is more matter than is visible in the Universe, or our understanding of gravity fails for low gravity regimes, or maybe a combination of these two possibilities.

In kinematics of spiral galaxies, this phenomenon is shown by the fact that rotation curves do not decline in a Keplerian way $\left(V \propto r^{-1 / 2}\right)$, as would be expected from the distribution of visible matter. Instead, the outer gradient of the rotation curve shows a well-defined distribution of values, always higher than $-1 / 2$ and well described by the Universal Rotation Curve (Persic, Salucci \& Stel 1996, Salucci et al. 2007). This is often interpreted as the effect of an additional component in spiral galaxies, the dark matter halo. The currently favoured cosmological framework, $\Lambda$ Cold Dark Matter $(\Lambda \mathrm{CDM})$, explains the observations at large scales in the universe (e.g. the power spectrum of the cosmic microwave background, Komatsu et al. 2011), but on galaxy scales the dark matter density distribution predicted by $\Lambda$ CDM (Navarro et al. 1996) is at odds with observations (e.g. Gentile et al. 2004, de Blok 2010). Possible solutions to reconcile $\Lambda$ CDM with observations include the effect of baryonic physics on the distribution of dark matter (e.g. Governato et al. 2012) or the considering a particle that is "warm" instead of "cold" (e.g. Benson et al. 2012) but consensus is very far from being reached on how plausible and realistic these effects are, and most of all

\section{Modified Newtonian Dynamics (MOND)}

An alternative explanation to dark matter in galaxies is that below a certain gravitational acceleration $a_{0} \sim 1.2 \times 10^{-8} \mathrm{~cm} \mathrm{~s}^{-2}$ the effective gravitational force is higher than expected in Newtonian gravity (MOND, Modified Newtonian Dynamics, Milgrom 1983, see Famaey \& McGaugh 2012 for a review).

In MOND, the gravitational acceleration $\vec{g}$ is derived from the Newtonian acceleration $\overrightarrow{g_{\mathrm{N}}}$ via the following:

$$
\vec{g}=\overrightarrow{g_{\mathrm{N}}} / \mu\left(|g| / a_{0}\right)
$$

where $a_{0} \sim 1.2 \times 10^{-8} \mathrm{~cm} \mathrm{~s}^{-2}$ (Begeman, Broeils \& Sanders 1991), and $\mu(x)$ is the interpolating function, whose asymptotic values are $\mu(x)=x$ for $x \ll 1$ and $\mu(x)=1$ for $x \gg 1$. Frequently used forms of the $\mu(x)$ function are the "simple" interpolating function (Famaey \& Binney 2005, Zhao \& Famaey 2006):

$$
\mu_{\text {simple }}(x)=\frac{x}{1+x}
$$

and the "standard" interpolating function (Milgrom 1983):

$$
\mu_{\text {standard }}(x)=\frac{x}{\sqrt{1+x^{2}}}
$$

MOND can explain the observations of various types of galaxies, e.g. state-of-the-art kinematics of nearby spiral galaxies (Gentile et al. 2011), of our own Galaxy (Famaey, Bruneton \& 

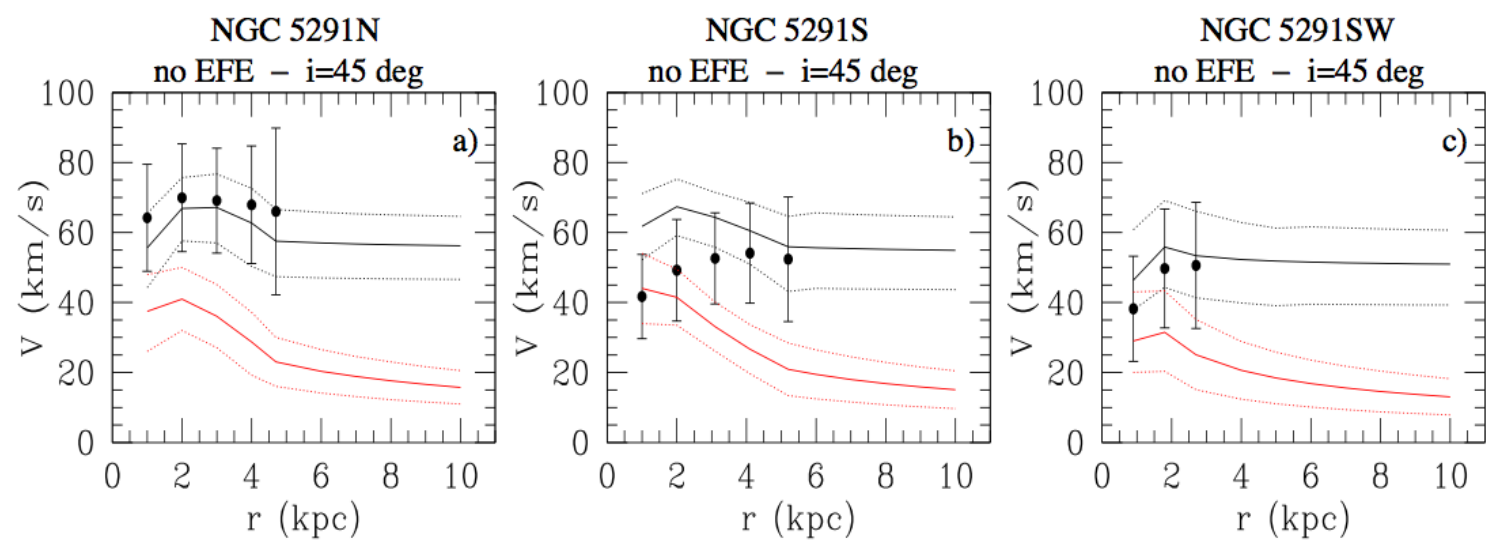

Figure 1: From Gentile et al. (2007): rotation curve data (full circles) of the 3 tidal dwarf galaxies observed by Bournaud et al. (2007). The lower (red) curves are the Newtonian contribution $V_{\text {bar }}$ of the baryons (and its uncertainty, indicated as dotted lines). The upper (black) curves are the MOND prediction and its uncertainty (dotted lines). These panels have implicit assumption (following Bournaud et al.) an inclination angle of 45 degrees.

Zhao 2007, McGaugh 2008, Bienaymé et al. 2009), and of early-type spiral galaxies (Sanders \& Noordermeer 2007). X-ray elliptical galaxies (Milgrom 2012), and most of all galaxy scaling relations such as the baryonic Tully-Fisher relation (McGaugh 2012) are very well accounted for in MOND.

The kinematics of tidal dwarf galaxies deserve some additional discussion. These galaxies are a by-product of the interaction between two spiral galaxies, when tidal tails are formed, and within these tidal tails some structures become self-gravitating: tidal dwarf galaxies. They are particularly important for dark matter studies because they should contain no (or little) dark matter (Barnes \& Hernquist 1992), contrary to other kinds of dwarf galaxies. In Gentile et al. (2007) we analysed the HI rotation curves published by Bournaud et al. (2007), who had made HI observations of the NGC 5291 system, using the VLA (Very Large Array): they found three tidal dwarf galaxies that are sufficiently resolved (NGC5291N, NGC5291S, and NGC5291SW) to allow a detailed investigation of their kinematics. Their kinematics reveals a mass discrepancy, which Bournaud et al. (2007) attribute to baryonic dark matter. However, in Gentile et al. (2007) we showed that, with zero free parameters, MOND can explain the kinematics of these three tidal dwarf galaxies (see Fig. 1).

However, we note that the MOND prescription is not sufficient to explain the observed discrepancy between visible and dynamical mass at scales larger than galaxies (e.g. Angus et al. 2007).

\section{Central surface densities}

In Donato et al. (2009) we studied the behaviour of the central surface density of dark matter, in a context where, contrary to Section 2, we assume Newtonian gravity and the presence of a dark matter halo. We have assumed that the dark matter halo in galaxies is described by the Burkert profile (Burkert 1995): 


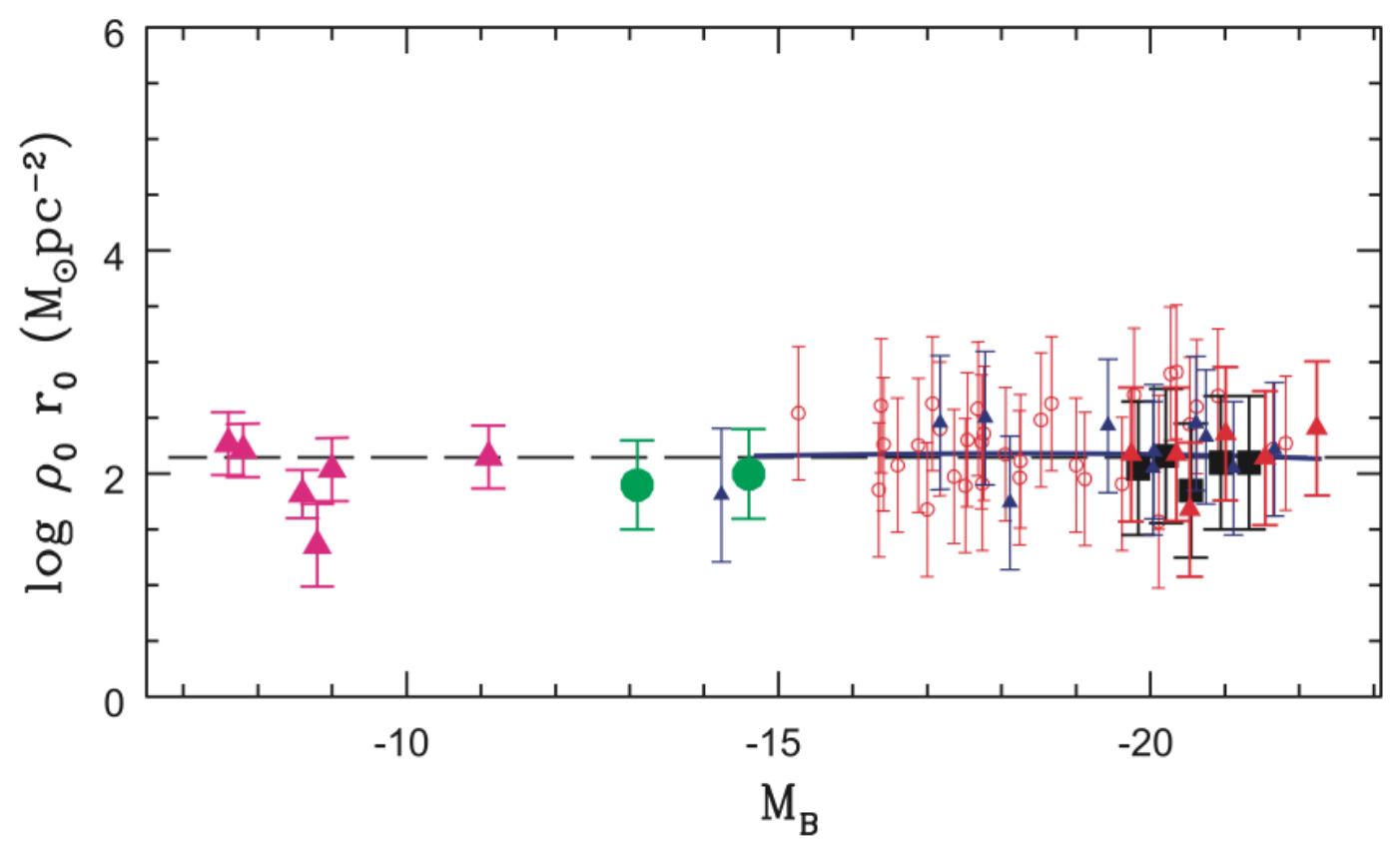

Figure 2: $\rho_{0} r_{0}$ as a function of galaxy magnitude for different subsamples of galaxies as shown in Donato et al. (2009). The Spano et al. (2008) data are shown as empty small red circles, and the other symbols are: the Universal Rotation Curve (solid blue line), two dwarf irregulars (NGC 3741 and DDO 47, full green circles), spirals and ellipticals investigated by weak lensing (black squares), dSphs (pink triangles), nearby spirals in THINGS (small blue triangles), and early-type spirals (full red triangles). The long-dashed line is the result of this work.

$$
\rho(r)=\frac{\rho_{0} r_{0}^{3}}{\left(r+r_{0}\right)\left(r^{2}+r_{0}^{2}\right)} .
$$

where $\rho_{0}$ is the central (approximately constant) density and $r_{0}$ is the core radius.

This dark matter density profile, when combined with the gaseous and stellar components, is found to reproduce very well the available kinematics of disk galaxies (e.g. Gentile et al., 2004). Moreover, it leads to estimates of the stellar disk mass in good agreement with the predictions from stellar population synthesis models (e.g. Salucci, Yegorova \& Drory 2008). The constant central surface density of dark matter in galaxies does not depend on which specific functional form of the (cored) density profile is assumed for the dark matter, i.e. whether one adopts a Burkert halo, a pseudo-isothermal halo (e.g. Oh et al. 2008) or other cored functional forms.

Spano et al. (2008) and Kormendy \& Freeman (2004) had noted that for a sample of spiral galaxies the product of the best-fit central density $\left(\rho_{0}\right)$ and the core radius $\left(r_{0}\right)$ is approximately constant or with a very weak dependence on other parameters such as luminosity. In Donato et al. (2009) we extended these results to a larger sample, with more galaxies and with a wider range of Hubble types and analysis methods, including weak lensing and stellar kinematics data. Fig. 2 illustrates the result of this investigation: over 14 galaxy magnitudes, the product $\rho_{0} r_{0}$ is consistent with a constant value. In addition to the Spano et al. (2008) galaxies, we made use of various datasets collected in the literature (see Donato et al. 2009 for details). 


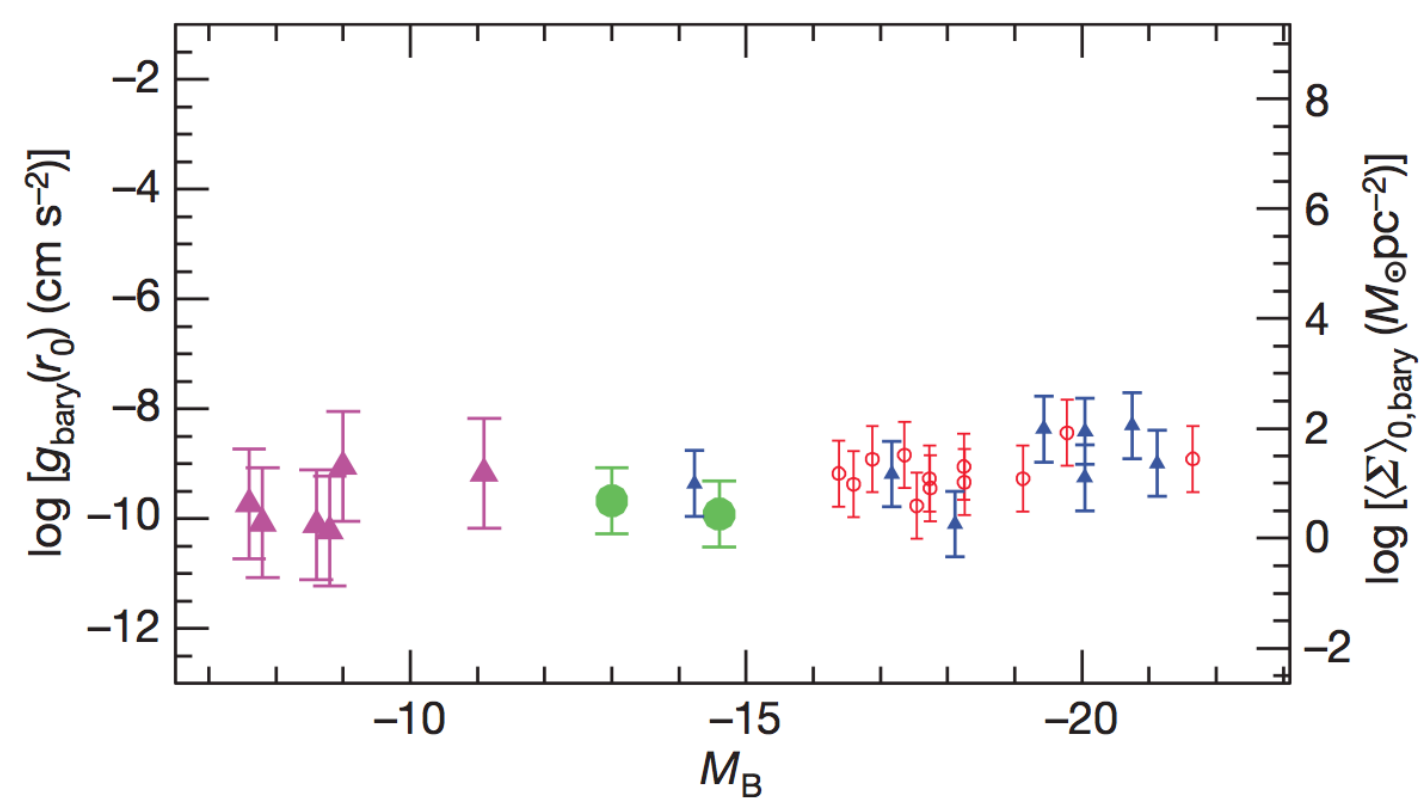

Figure 3: Universality of the average surface density (and gravity) of baryons within the halo core radius. $<\Sigma>_{0, b}$ and $g_{b}\left(r_{0}\right)$. plotted as a function of the B-band absolute magnitude of the galaxies, from Gentile et al. (2009). Symbols are like Fig. 2.

We find that the product $\mu_{0} \equiv \rho_{0} r_{0}$ is consistent with the following universal value:

$$
\log \left(\mu_{0} / \mathrm{M}_{\odot} \mathrm{pc}^{-2}\right)=2.15 \pm 0.2
$$

or, equivalently,

$$
\mu_{0}=140_{-30}^{+80} \mathrm{M}_{\odot} \mathrm{pc}^{-2}
$$

In Gentile et al. (2009) we interpreted this as the average dark matter surface density within $r_{0},<\Sigma>_{0, D M}$, being also constant: if we define it in terms of $M\left(<r_{0}\right)$, the enclosed mass within $r_{0}$, the average dark matter surface density is then: $\left\langle\Sigma>_{0, D M}=M\left(<r_{0}\right) /\left(\pi r_{0}^{2}\right) \sim 0.51 \rho_{0} r_{0}=\right.$ $0.51 \mu_{0}=72_{-27}^{+42} \mathrm{M}_{\odot} \mathrm{pc}^{-2}$. Apart from a constant factor, this is also equivalent to the gravitational acceleration generated by the dark matter halo at $r_{0}$, which thus is also universal: $g_{D M}\left(r_{0}\right)=G \pi<$ $\Sigma>_{0, D M}=3.2_{-1.2}^{+1.8} 10^{-9} \mathrm{~cm} \mathrm{~s}^{-2}$.

Can we say something about the average baryonic surface density within $r_{0}$ ? In a similar way to the dark matter surface density, we looked for a dependency with galaxy luminosity of $g_{b}\left(r_{0}\right)$, the gravitational acceleration generated by baryonc at $r_{0}$ (equivalent to average baryonic surface density within $r_{0},\left\langle\Sigma>_{0, b}=g_{b}\left(r_{0}\right) /(G \pi)\right)$. It turns out that, also in the case of baryons, these values are consistent with a universal value: $g_{b}\left(r_{0}\right)=5.7_{-2.8}^{+3.8} 10^{-10} \mathrm{~cm} \mathrm{~s}^{-2}$, see Fig. 3 .

\section{References}

[1] Angus, G. W., Shan, H. Y., Zhao, H. S., \& Famaey, B. 2007, ApJ, 654, L13

[2] Begeman, K. G., Broeils, A. H., Sanders, R. H., 1991, MNRAS, 249, 523

[3] Benson, A. J., Farahi, A., Cole, S., et al. 2012, MNRAS in press (arXiv:1209.3018) 
[4] Bienaymé, O., Famaey, B., Wu, X., Zhao, H. S., \& Aubert, D. 2009, A\&A, 500, 801

[5] Bournaud, F., et al. 2007, Science, 316, 1166

[6] Burkert, A., 1995 ApJ, 447, L25

[7] de Blok, W. J. G. 2010, Advances in Astronomy, 2010, 789293

[8] Donato, F., Gentile, G., Salucci, P., et al. 2009, MNRAS, 397, 1169

[9] Famaey, B., \& Binney, J. 2005, MNRAS, 363, 603

[10] Famaey, B., Bruneton, J.-P., \& Zhao, H. 2007, MNRAS, 377, L79

[11] Famaey, B., \& McGaugh, S. S. 2012, Living Reviews in Relativity, 15, 10

[12] Gentile, G., Salucci, P., Klein, U., Vergani, D., Kalberla, P., 2004, MNRAS, 351, 903

[13] Gentile, G., Famaey, B., Combes, F., Kroupa, P., Zhao, H. S., Tiret, O. 2007, A\&A, 472, L25

[14] Gentile, G., Famaey, B., Zhao, H., \& Salucci, P. 2009, Nature, 461, 627

[15] Gentile, G., Famaey, B., \& de Blok, W. J. G. 2011, A\&A, 527, A76

[16] Governato, F., Zolotov, A., Pontzen, A., et al. 2012, MNRAS, 422, 1231

[17] Komatsu, E., Smith, K. M., Dunkley, J., et al. 2011, ApJS, 192, 18

[18] Kormendy, J., Freeman, K. C. 2004, IAU Symposium, Sydney, Astronomical Society of the Pacific, 220,377

[19] McGaugh, S. S. 2008, ApJ, 683, 137

[20] McGaugh, S. S. 2012, AJ, 143, 40

[21] Milgrom, M., 1983, ApJ, 270, 365

[22] Milgrom, M. 2012, Physical Review Letters, 109, 131101

[23] Navarro, J.F., Frenk, C.S, White, S.D.M., 1996, ApJ, 462, 563

[24] Oh, S.-H., de Blok, W. J. G., Walter, F., Brinks, E., \& Kennicutt, R. C., Jr. 2008, AJ, 136, 2761

[25] Persic, M., Salucci, P., Stel, F., 1996 MNRAS, 281, 27

[26] Salucci, P., Lapi, A., Tonini, C., et al. 2007, MNRAS, 378, 41

[27] Salucci P., Yegorova I. A., Drory N., 2008, MNRAS, 388, 159

[28] Sanders, R. H., \& Noordermeer, E. 2007, MNRAS, 379, 702

[29] Spano, M. et al., 2008, MNRAS, 383, 297

[30] Zhao, H. S., \& Famaey, B. 2006, ApJ, 638, L9 